\title{
Weak first-order orientational transition in the Lebwohl-Lasher model for liquid crystals
}

\section{Zhang, Zhengping; Mouritsen, Ole G.; Zuckermann, Martin J.}

\section{Published in:}

Physical Review Letters

Link to article, DOI:

10.1103/PhysRevLett.69.2803

Publication date:

1992

Document Version

Publisher's PDF, also known as Version of record

Link back to DTU Orbit

Citation (APA):

Zhang, Z., Mouritsen, O. G., \& Zuckermann, M. J. (1992). Weak first-order orientational transition in the Lebwohl-Lasher model for liquid crystals. Physical Review Letters, 69(19), 2803-2806.

https://doi.org/10.1103/PhysRevLett.69.2803

\section{General rights}

Copyright and moral rights for the publications made accessible in the public portal are retained by the authors and/or other copyright owners and it is a condition of accessing publications that users recognise and abide by the legal requirements associated with these rights.

- Users may download and print one copy of any publication from the public portal for the purpose of private study or research.

- You may not further distribute the material or use it for any profit-making activity or commercial gain

- You may freely distribute the URL identifying the publication in the public portal 


\title{
Weak First-Order Orientational Transition in the Lebwohl-Lasher Model for Liquid Crystals
}

\author{
Zhengping Zhang, ${ }^{(1)}$ Ole G. Mouritsen, ${ }^{(2)}$ and Martin J. Zuckermann ${ }^{(1)}$ \\ (1) Centre for the Physics of Materials, Department of Physics, McGill University, Rutherford Building, \\ 3600 University Street, Montréal, Québec, Canada H3A 2T8 \\ ${ }^{(2)}$ Canadian Institute of Advanced Research and Department of Physical Chemistry, The Technical University of Denmark, \\ Building 206, DK-2800 Lyngby, Denmark
}

(Received 12 June 1992)

\begin{abstract}
The nature of the orientational phase transition in the three-dimensional Lebwohl-Lasher model of liquid crystals has been studied by computer simulation using reweighting techniques and finite-size scaling analysis. Unambiguous numerical evidence is found in favor of a weak first-order transition and the presence of pseudospinodal points, $T_{ \pm}^{*}$, which are extremely close to the equilibrium transition temperature, $\left|T_{c}-T_{ \pm}^{*}\right| / T_{c} \lesssim 0.5 \times 10^{-3}$, in good agreement with experimental data for the nematic-isotropic transition.

PACS numbers: $64.70 . \mathrm{Md}, 64.60 . \mathrm{Cn}$
\end{abstract}

The Lebwohl-Lasher model [1] is the lattice version of the Maier-Saupe model [2] of an anisotropic liquid in which the molecules are coupled by the Hamiltonian

$$
\mathcal{H}=-\epsilon \sum_{i, j} P_{2}\left(\cos \theta_{i j}\right),
$$

where $P_{2}\left(\cos \theta_{i j}\right)=\frac{1}{2}\left(3 \cos ^{2} \theta_{i j}-1\right), \theta_{i j}$ is the angle between the axes of rotor molecules at nearest-neighbor sites $i$ and $j$, and $\epsilon$ is a coupling parameter. The LebwohlLasher model neglects the coupling between the translational variables and the orientational variables which is present in a real nematogen and it is therefore an appropriate model for orientational ordering in a solid whereas it is leaving out important properties of liquid crystals. Still it is believed that this model can reveal some of the essential transitional properties of liquid crystals near the nematic-isotropic phase transition. The orientational order in the Lebwohl-Lasher model is characterized by a second-rank tensor order parameter, $\mathbf{Q}$. Within the Landau-de Gennes theory [3] the free energy of the model is expanded in components of this order parameter as $F=F_{0}+\frac{1}{2} A(T) Q_{\alpha \beta} Q_{\beta \alpha}+\frac{1}{3} B(T) Q_{\alpha \beta} Q_{\beta \gamma} Q_{\gamma \alpha}+\mathcal{O}\left(Q^{4}\right)$. $F$ contains a third-order invariant which implies that the model should exhibit a first-order transition at a temperature $T_{c}$. From the mean-field solution to the Maier-Saupe model it can be estimated that the spinodal temperature $T_{-}^{*}$, which marks the stability limit of the isotropic phase, is displaced relative to the equilibrium transition temperature by $\left(T_{c}-T_{-}^{*}\right) / T_{c} \sim 10^{-1}$ which is almost 2 orders of magnitude larger than typical experimental values $[3-5]$.

The nature and the properties of the orientational phase transition in the Lebwohl-Lasher model have been under active investigation for a considerable time $[1$, 6-10]. No unambiguous evidence for the first-order nature of the transition has as yet been presented in a calculation which fully allows for fluctuations and it has remained unclear to what extent the model may de- scribe the close proximity of the experimentally observed stability limit of the isotropic phase to the transition point. In this Letter, we have exploited modern numerical techniques of analyzing phase transitions via a finite-size scaling analysis to give conclusive numerical evidence for the presence of a very weak first-order transition in the three-dimensional Lebwohl-Lasher model. The transition is possibly weaker than the transition in the three-dimensional three-state Potts model [11]. Furthermore, by determining the density of states for the model and from the density of states generating the free energy in the metastable and unstable regimes, we are able to determine an upper limit of the distance of the pseudospinodal points from the transition to be $\left|T_{c}-T_{ \pm}^{*}\right| / T_{c} \lesssim 0.5 \times 10^{-3}$. This result is in good agreement with experimental data for room-temperature nematogens [3-5].

We have investigated the orientational transition in the Lebwohl-Lasher model on a simple cubic lattice with periodic boundary conditions by performing a series of extensive Monte Carlo computer-simulation calculations of the temperature dependence of the internal energy, the orientational (nematic) order parameter, and the corresponding response functions, i.e., the specific heat, $C(T, L)$, and the ordering susceptibility, $\chi(T, L)$. The calculations have been performed systematically for a number of different lattice sizes, $L^{3}$. In order to obtain maximum statistical accuracy, the Ferrenberg-Swendsen reweighting technique [12] has been used. This technique works on the level of density of states or distribution functions from which the relevant part of the free energy can be derived. A finite-size scaling analysis along the lines suggested by Lee and Kosterlitz [13,14] has then been applied to the data to assess the nature of the phase transition. The Lee-Kosterlitz method constitutes an unambiguous method of numerically detecting a first-order transition [14].

The value of orientational order parameter is deter- 
mined as

$$
\left\langle P_{2}\right\rangle=L^{-d}\left\langle\sum_{i}\left(\frac{3}{2} \cos ^{2} \theta_{i}-\frac{1}{2}\right)\right\rangle,
$$

where $\theta_{i}$ is the angle between the axis of the $i$ th rotor molecule and the nematic director. Because of the nonbroken continuous symmetry of the orientational ordering, the direction of the nematic director varies and has to be determined during the simulation in order to calculate $\left\langle P_{2}\right\rangle$. This is facilitated by diagonalization of the tensor order parameter $\mathbf{Q}$. The instantaneous value of the order parameter is then given by the largest eigenvalue, $\lambda$, of $\mathbf{Q}[8]$. The thermodynamic order parameter is obtained as the average, $\langle\lambda\rangle \equiv\left\langle P_{2}\right\rangle_{\lambda}$.

A first-order phase transition is characterized by discontinuities in the first derivatives of the free energy. This results in the thermodynamic limit in singularities at the transition for the specific heat $C(T)$ and for the ordering susceptibility $\chi(T)$. In a finite system, however, the transition region is broadened and the peaks in $C(T)$ and $\chi(T)$ are finite and their height increases with increasing linear lattice size $L$ [15]. Furthermore, the location of the maxima varies in a size-dependent manner. The maxima grow as $L^{d}$ in $d$ dimensions, e.g., $\chi_{\max }(L) \sim L^{d}$.

The Monte Carlo simulations are performed in three steps. First, simulations are performed to determine as accurately as possible the positions of the peaks in $C(T, L)$ and $\chi(T, L)$. Second, extensive simulations are performed very close to the transition for different values of $L$. These simulations typically involve $(1-2) \times 10^{6}$ Monte Carlo steps per site. Third, the reweighting technique of Ferrenberg and Swendsen [12] is used to calculate the distribution function $\mathcal{P}(\lambda, T, L)$ for the order parameter for a range of temperatures in the transition region. From $\mathcal{P}(\lambda, T, L)$ the response functions can be calculated in the transition region. A finite-size scaling analysis can then be applied to the data. As an example, Fig. 1 shows data for the susceptibility, $\chi(T, L)$, and how the maximum, $\chi_{\max }(L)$, scales with system size. The expected scaling relation for a first-order transition is found to hold, $\chi_{\max }(L) \sim L^{d}$.

The order of the phase transition can be examined more closely by the method of Lee and Kosterlitz [13, 14]. This method involves a calculation of the free energy as a function of the order parameter at the transition using the probability distribution $\mathcal{P}(\lambda, T, L)$. The free-energy-like quantity $\mathcal{F}(\lambda, T, L)$ defined by [14]

$$
\mathcal{F}(\lambda, T, L) \sim-\ln \mathcal{P}(\lambda, T, L)
$$

differs from the bulk free energy by a temperature- and $L$-dependent additive quantity. However, at fixed $T$ and $L$, the shape of $\mathcal{F}(\lambda, T, L)$ is identical to that of the bulk free energy and furthermore $\mathcal{F}(\lambda, T, L)-\mathcal{F}\left(\lambda^{\prime}, T, L\right)$ is a correct measure of free-energy differences. At a firstorder transition, $\mathcal{F}(\lambda, L)$ has pronounced double minima corresponding to two coexisting phases at $\lambda=\lambda_{1}$ and

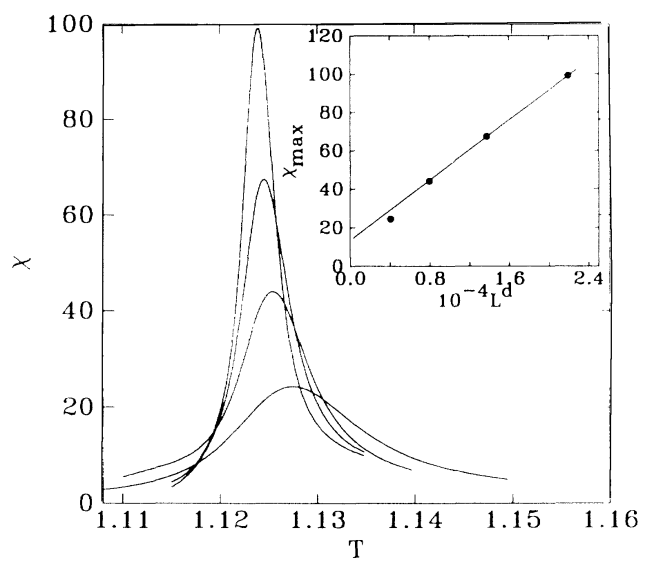

FIG. 1. Ordering susceptibility, $\chi(T)$ (in units of $\epsilon^{-1}$ ), as a function of temperature $T$ (in units of $\epsilon / k_{B}$, for four different lattice sizes, $L^{3}=16^{3}, 20^{3}, 24^{3}$, and $28^{3}$. Inset: The finite-size scaling behavior of the maximum, $\chi_{\max }(L)$, of the susceptibility.

$\lambda=\lambda_{2}$ separated by a barrier, $\Delta \mathcal{F}(L)$, with a maximum at $\lambda_{\max }$ corresponding to an interface between the two phases. The height of the barrier measures the interfacial free energy between the two coexisting phases and is given by $\Delta \mathcal{F}(L)=\mathcal{F}\left(\lambda_{\max }, L\right)-\mathcal{F}\left(\lambda_{1}, L\right) \sim L^{d-1}$. Therefore, at a first-order transition, $\Delta \mathcal{F}(L)$ increases monotonically with $L$. The finding of such an increase is an unambiguous sign of a first-order transition [14]. In contrast, $\Delta \mathcal{F}(L)$ approaches a constant at a continuous transition and vanishes in the absence of a transition [13, 16]. We have measured the transition temperature in the finite system in three different ways: from the position of the maximum in the specific heat, $T_{c}^{C}(L)$, from the position of the maximum in the susceptibility, $T_{c}^{\chi}(L)$, and from the criterion that the two minima in the free energy are equally deep, $T_{c}^{\mathcal{F}}(L)$. In Fig. 2 are shown the data for the free energy for different system sizes determined at temperatures, $T_{c}^{\mathcal{F}}(L)$. The finite-size behavior is very clear: As the system size is increased, a doublewell structure develops in the free energy. This is conclusive numerical evidence in favor of a first-order transition. Since the barrier between the two minima is only well pronounced for the two larger system sizes, we have insufficient data to assess the scaling behavior of the barrier height. This shows that the first-order transition is very weak and in this sense it is considerably weaker than the first-order transitions in the three-dimensional threestate Potts model [11] and the two-dimensional five-state Potts model [13] which are notoriously known to be weak first-order transitions.

In Fig. 3 are shown the results for the different measures of the finite-system phase transition temperature and how they scale with system size. For finite systems, the different measures of the transition temperature vary, but they should tend to the same value in the thermody- 


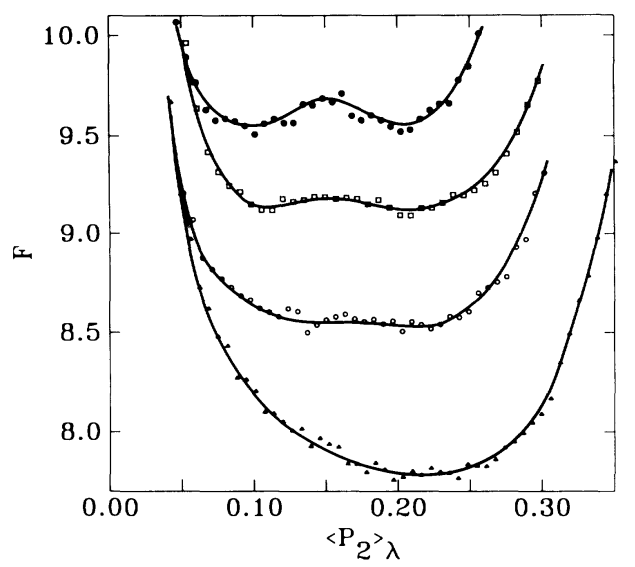

FIG. 2. Free energy $\mathcal{F}$ (in units of $\epsilon$ ) as a function of orientational order $\left\langle P_{2}\right\rangle_{\lambda}$ for four different lattice sizes, $L^{3}=$ $16^{3}(\Delta), 20^{3}(\circ), 24^{3}(\square)$, and $28^{3}(\bullet)$. The solid lines are guides to the eye. For the sake of clarity, the different sets of data are displaced vertically.

namic limit, $L \rightarrow \infty$. This is indeed the case as shown in Fig. 3, which furthermore demonstrates that the expected finite-size scaling relation, $T_{c}(L)-T_{c} \sim L^{-d}$, at a first-order phase transition is in effect. From extrapolation to the thermodynamic limit we obtain our estimate of the equilibrium first-order transition temperature, $T_{c}=(1.1232 \pm 0.0001) \epsilon / k_{B}$. The value of the transition enthalpy, $\Delta H=\int C(T) d T$, can be estimated from the finite-size scaling behavior of the specific heat and we find $\Delta H \simeq(0.20 \pm 0.04) \epsilon$.

We now turn to a discussion of the pretransitional effects [3]. The very weak appearance of the first-order orientational transition in the Lebwohl-Lasher model suggests that the fluctuations of the orientational order in the transition region are controlled by nearby criticalpoint-like singularities which lie at the termini of the metastable branches of the free energy. The use of distribution functions, such as $\mathcal{P}(\lambda, T, L)$, and the derived free-energy functions, $\mathcal{F}(\lambda, T, L)$ in Eq. (3), permit a detailed study of the limits of stability of both the orientationally ordered and the isotropic phase. These limits of stability are not rigorously defined for a system with short-range interactions [17]. Hence we refer here to these limits, $T_{ \pm}^{*}$, as pseudospinodal points rather than spinodal points. $T_{-}^{*}$ denotes the stability limit of the isotropic phase in the orientationally ordered phase and $T_{+}^{*}$ denotes the stability limit of the orientationally ordered phase in the isotropic phase. The numerical data from which $T_{ \pm}^{*}(L)$ can be assessed are shown in Fig. 4. In this figure the free energy is displayed for the largest system size studied, $L^{3}=28^{3}$, for different values of the temperature around the equilibrium transition temperature. The figure shows the "classic" behavior of a free-energy function near a first-order transition as it is usually presented from the Landau theory [18]. The pseudospinodal

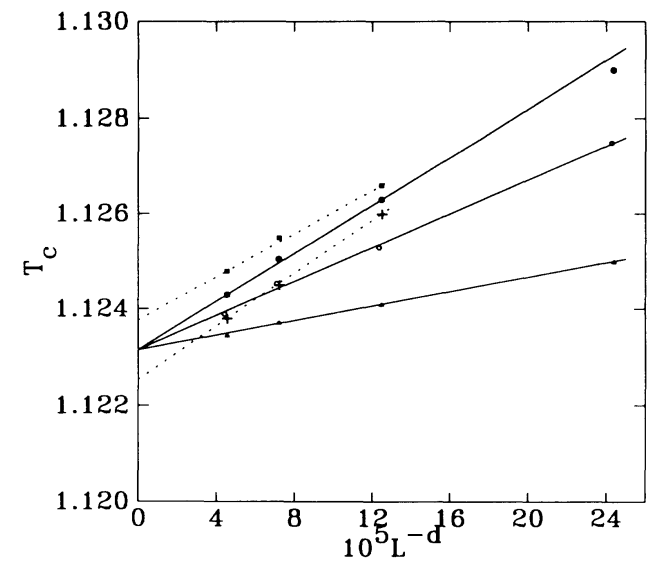

FIG. 3. Finite-size scaling behavior shown for three different measures of the finite-system transition temperature $T_{c}(L): T_{c}^{\mathcal{F}}(L)(\bullet), T_{c}^{\chi}(L)(\circ), T_{c}^{C}(L)(\mathbf{\Delta})$. The estimates of the finite-size pseudospinodal points are also shown, $T_{-}^{*}(L)$ $(+)$ and $T_{+}^{*}(L)(\square)$. Extrapolations to the thermodynamic limits, $T_{c}(\infty)$ and $T_{ \pm}^{*}(\infty)$, are denoted by solid and dashed lines, respectively.

points in the finite system are estimated as the temperatures where the second, local minima of the free energy vanish as the temperature is changed away from the transition temperature. The pseudospinodal points, $T_{ \pm}^{*}$, in the thermodynamic limit are determined from an extrapolation as indicated in Fig. 3. We note that we have

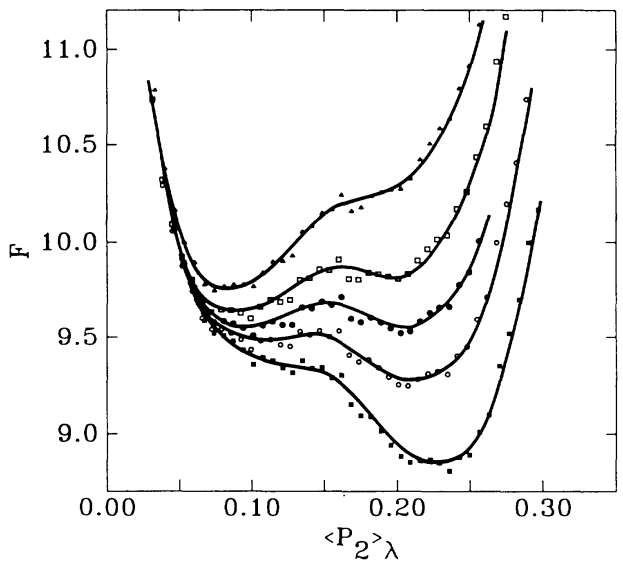

FIG. 4. Free energy $\mathcal{F}$ (in units of $\epsilon$ ) as a function of orientational order $\left\langle P_{2}\right\rangle_{\lambda}$ for a lattice with $L^{3}=28^{3}$ sites. Results are given corresponding to five different temperatures near the orientational phase transition. From bottom to top the temperatures (in units of $\epsilon / k_{B}$ ) are $1.1238(\square), 1.1241$ (०), $1.1243(\bullet), 1.1245(\square)$, and $1.1248(\Delta)$. The middle curve corresponds to the finite-size equilibrium transition temperature $T_{c}(L)^{\mathcal{F}}$. Pairwise the sets of curves away from the transition correspond to metastability and pseudospinodal behavior, respectively. The solid lines are guides to the eye. For the sake of clarity, the different sets of data are displaced vertically. 
no rigorous basis for the finite-size scaling behavior of $T_{ \pm}^{*}(L)$. Hence we want to consider our estimates of $T_{ \pm}^{*}$ to denote the maximal displacement from the transition. Our result from Fig. 3 is that the pseudospinodal points are extremely close to the equilibrium transition temperature, $\left|T_{c}-T_{ \pm}^{*}\right| / T_{c} \lesssim 0.5 \times 10^{-3}$.

The powerful reweighting technique by Ferrenberg and Swendsen [12] makes it feasible to generate numerical data for the free energy, cf. Figs. 2 and 4, which give detailed insight into the nonequilibrium properties of a system which undergoes such a weak first-order transition as the three-dimensional Lebwohl-Lasher model. This is probably the reason that earlier numerical work on systems as large as $30^{3}$ molecules [7] on the model using more conventional techniques involving distribution functions had difficulty in matching the two free-energy minima. In the work by Fabbri and Zannoni [7] the stability limit of the isotropic phase was investigated by an extrapolation analysis of an Ornstein-Zernike expression for the pair correlation function. The result found for a $30^{3}$ system, $\left(T_{c}-T_{-}^{*}\right) / T_{c} \simeq 3 \times 10^{-3}$, is in orderof-magnitude agreement with the value obtained in the present calculation which indicates that the assumptions underlying the work in Ref. [7] are resonable [19].

Finally, we wish to compare our results to experimental data for the nematic-isotropic transition in liquid crystals. It has been found [3-5] for a large series of roomtemperature nematogens that the transition enthalpy and the relative stability limit of the isotropic phase are only slightly sensitive to the material in question. Hence it is pertinent to compare results from the simple and parameterless Lebwohl-Lasher model with experimental data. As a specific example we refer here to the liquid crystal octylcyanobiphenyl (8CB) $[4,5]$ with the following experimental transitional properties, $T_{c}=40.8^{\circ} \mathrm{C}$ and $\Delta H=612 \mathrm{~J} / \mathrm{mol}$. From the experimental transition temperature we can determine the value of the energy parameter $\epsilon$, which leads to $\Delta H=460 \mathrm{~J} / \mathrm{mol}$ for the Lebwohl-Lasher model. Since nematics usually cannot be supercooled [5], the experimental determination of the stability limit of the isotropic phase is not very accurate since it is obtained by extrapolation of the equilibrium data for the susceptibility or the specific heat from the isotropic phase into the nematic phase. The experimental values $[4,5]$ quoted for $\left(T_{c}-T_{-}^{*}\right) / T_{c}$ lie in the range $\sim(0.2-3) \times 10^{-3}$ in good agreement with our results from the Lebwohl-Lasher model. Hence it appears that the Lebwohl-Lasher model is quite successful in describing experimental data for those transitional properties which are not very sensitive to the nature of the material.
This work was supported by FCAR du Quebec under a centre and team grant, by NSERC of Canada, and by the Danish Natural Science Research Council under Grant No. 11-7785. The Danish Research Academy supported M.J.Z. and Z.Z. during their stay in Denmark under Grant No. S910015. UNI.C is thanked for access to the CM-200 during the early stage of this work.

[1] P. A. Lebwohl and G. Lasher, Phys. Rev. A 6, 426 (1972); 7, 2222(E) (1973).

[2] W. Maier and A. Saupe, Z. Naturforsch. 14A, 882 (1959); 15A, 287 (1960).

[3] P. G. de Gennes, The Physics of Liquid Crystals (Clarendon, Oxford, 1974).

[4] T. W. Stinson and J. D. Litster, Phys. Rev. Lett. 25, 503 (1970).

[5] J. Thoen, H. Marynissen, and W. Van Dael, Phys. Rev. A 26, 2886 (1982).

[6] G. R. Luckhurst and P. Simpson, Mol. Phys. 47, 251 (1982).

[7] U. Fabbri and C. Zannoni, Mol. Phys. 58, 763 (1986)

[8] C. Zannoni, J. Chem. Phys. 84, 424 (1986).

[9] F. Biscarini, C. Zannoni, C. Chiccoli, and P. Pasini, Mol. Phys. 73, 439 (1991).

[10] D. J. Cleaver and M. P. Allen, Phys. Rev. B 43, 1918 (1991).

[11] J. Lee and J. M. Kosterlitz, Phys. Rev. B 43, 1268 (1991).

[12] A. M. Ferrenberg and R. H. Swendsen, Phys. Rev. Lett. 61, 2635 (1988).

[13] J. Lee and J. M. Kosterlitz, Phys. Rev. Lett. 65, 137 (1990).

[14] J. Lee and J. M. Kosterlitz, Phys. Rev. B 43, 3265 (1991).

[15] M. S. S. Challa, D. P. Landau, and K. Binder, Phys. Rev. B 34, 1841 (1986).

[16] Z. Zhang, M. Laradji, H. Guo, O. G. Mouritsen, and M. J. Zuckermann, Phys. Rev. A 45, 7560 (1992).

[17] J. D. Gunton, M. San Miguel, and P. S. Sahni, in Phase Transitions and Critical Phenomena, edited by C. Domb and J. L. Lebowitz (Academic, New York, 1983), Vol. 8, p. 267.

[18] H. E. Stanley, Introduction to the Theory of Phase Transitions and Critical Phenomena (Clarendon, Oxford, 1971).

[19] The transition temperature obtained from the work in Ref. [7], $T_{c}=(1.1232 \pm 0.0006) \epsilon / k_{B}$ was basically obtained from an analysis of the finite-size behavior of the specific heat. This value is identical to the value obtained in the present work. As shown in Fig. 3, the size dependence of $T_{c}^{C}(L)$ is less pronounced in the scaling regime than of $T_{c}^{\chi}(L)$ and $T_{c}^{\mathcal{F}}(L)$ and hence very accurate values of the transition temperature can be obtained directly from specific-heat data for large systems as done in Ref. [7]. 